\title{
Elemental abundance analyses with Complejo Astronómico El Leoncito REOSC echelle spectrograms. I. $\kappa$ Cancri, HR 7245, and $\xi$ Octantis
}

\author{
O.I. Pintado ${ }^{1, \star}$ and S.J. Adelman ${ }^{2, \star \star}$ \\ 1 Complejo Astronómico El Leoncito, CC 468, 5400 San Juan, Argentina \\ 2 Department of Physics, The Citadel, 171 Moultrie Street, Charleston, SC 29409, U.S.A.
}

Received November 14, 1995; accepted January 19, 1996

\begin{abstract}
Elemental abundances are derived for three sharp-lined stars $\kappa$ Cnc, HR 7245, and $\xi$ Oct using REOSC echelle spectrograms obtained at CASLEO. Comparisons are made with published equivalent widths. The derived abundances for $\kappa \mathrm{Cnc}$ and $\xi$ Oct are slightly larger than those obtained with other high dispersion spectrographs. HR 7245 exhibits a pattern of abundance anomalies similar to other Mercury-Manganese stars. The spectra of the two HgMn stars in the $\lambda \lambda 4640-5100$ region exhibit an interesting and useful variety of lines which can be used to supplement analyses of the photographic region.
\end{abstract}

Key words: stars: abundances - stars: chemically peculiar - stars: $\kappa$ Cnc - HR $7245-\xi$ Oct

\section{Introduction}

The Southern Hemisphere has many bright early type stars. Those towards the South Celestial Pole remain relatively unexplored compared with similar stars in the Northern Hemisphere. They include examples of both common and rare types. In selecting stars for space observations astronomers have often been biased in their choices as the well examined stars of a given type are often those accessible from observatories in the Northern Hemisphere. This paper, which we hope will be the first in a series, examines the sharp-lined Southern Hemispheric star $\xi$ Octantis as well as $\kappa$ Cancri and HR 7245 which are in the equatorial region.

$\kappa$ Cnc (= HD $79316=\mathrm{HR} 3623)$ is a MercuryManganese (Hg-Mn) star analyzed most recently by Adelman (1987) using coadded Dominion Astrophysical Observatory (DAO) IIaO spectrograms. It is one of the hottest members of this class and a single-lined spectroscopic binary. Its apparent rotational velocity is $6 \mathrm{~km} \mathrm{~s}^{-1}$. HR 7245 (= HD 178065) is a sharp-lined Mercury star which most

Send offprint requests to: S.J. Adelman

${ }^{\star}$ Member of Carrera del Investigador del Consejo Nacional de Investigaciones Científicas y Técnicas

${ }^{\star \star}$ Visiting Astronomer, Complejo Astronómico El Leoncito operated under agreement between the Consejo Nacional de Investigaciones Científicas y Técnicas de la República Argentina and the National Universties of La Plata, Córdoba and San Juan recently analyzed by Zakharova (1994). Guthrie (1984, 1985 ) found that its elemental abundances are similar to those of other $\mathrm{HgMn}$ stars with similar temperatures. Its $Y$ abundance is among the least overabundant for the class. It is a single-lined spectroscopic binary with a period of 6.87 days.

The sharp-lined B6 IV star $\xi$ Oct $(=$ HR $8663=\mathrm{HD}$ 215573) has elemental abundances which are typical for those of normal B main-sequence band stars (Adelman et al. 1993). On average the derived values are 0.28 dex less than solar. It is one of the very few known sharp-lined middle B stars. If its true rotational velocity is typical of similar stars, then we are looking almost directly at one of its rotational poles.

The spectra were obtained at the Complejo Astronómico El Leoncito (CASLEO) located at latitude $-31^{\circ} 47^{\prime} 57^{\prime \prime}$, longitude $4 \mathrm{~h} 37 \mathrm{~m} 12 \mathrm{~s}$ West at an altitude of $2550 \mathrm{~m}$. We used the $2.15-\mathrm{m}$ telescope, a twin to the similarly sized telescope at Kitt Peak National Observatory (KPNO), and a REOSC echelle spectrograph, which is on loan from the Institute Astrophysique de Liège, Belgium and a Tek 1024 CCD. A grating with 1200 lines $\mathrm{mm}^{-1}$ was used as a cross disperser. The resolution is $0.10 \AA$ pixel $^{-1}$ and the spectral range is about $650 \AA$ per CCD exposure. A Th-Ar lamp was used for the wavelength calibration.

As this paper contains the first analyses from this telescope-instrument combination, we decided to study three stars for which some equivalent widths have been 
Table 1. He/H values for $\xi$ Oct and HR 7245

\begin{tabular}{lrrrrrr}
\hline & \multicolumn{3}{c}{$\xi$ Oct } & \multicolumn{3}{c}{ HR 7245 } \\
$\lambda(\AA)$ & $W_{\lambda}(\mathrm{m} \AA)$ & $\mathrm{He} / \mathrm{H}$ & $W_{\lambda}(\mathrm{m} \AA)$ & $\mathrm{He} / \mathrm{H}$ & $W_{\lambda}(\mathrm{m} \AA)$ & $\mathrm{He} / \mathrm{H}$ \\
\hline 3867 & 55 & $0.045:$ & 70 & 0.060 & $\ldots$ & $\ldots$ \\
4009 & $\ldots$ & $\ldots$ & 156 & 0.063 & $\ldots$ & $\ldots$ \\
4026 & 704 & 0.082 & 618 & 0.063 & 163 & 0.015 \\
4121 & 72 & 0.062 & 81 & 0.074 & $\ldots$ & $\ldots$ \\
4143 & $\ldots$ & $\ldots$ & 310 & 0.108 & 86 & 0.040 \\
4169 & $\ldots$ & $\ldots$ & 33 & 0.093 & $\ldots$ & $\ldots$ \\
4388 & 294 & 0.074 & 288 & 0.071 & $\ldots$ & $\ldots$ \\
4438 & $\ldots$ & $\ldots$ & 35 & 0.057 & $\ldots$ & $\ldots$ \\
4472 & $\ldots$ & $\ldots$ & 671 & 0.117 & 155 & 0.020 \\
4713 & $\ldots$ & $\ldots$ & $\ldots$ & $\ldots$ & 18 & 0.010 \\
4921 & $\ldots$ & $\ldots$ & $\ldots$ & $\ldots$ & 56 & 0.020 \\
& & & & & & \\
Average & & 0.073 & & 0.078 & & 0.021 \\
\hline
\end{tabular}

published. We will be working to understand any differences in the line profiles (and equivalent widths) such as those produced by the instrumental profiles including scattered light. CASLEO is a relatively high dry site which tends to minimize telluric lines. This is especially important in the red and infrared. For equatorial stars of interest, we can extend the analyses of stars from Northern Hemispheric observatories, for example, the DAO, whose coudé spectrograph is optimized for $\lambda \lambda 3800-5000$, into the yellow and red.

\section{Reduction of spectrograms}

The spectra reductions were made using IRAF $2.10^{1}$. With bias and flat fields we obtained a combined flat field which was used to divide the spectra to remove the pixel-to-pixel variations. The extraction was performed with APALL and the wavelength calibration with IDENTIFY and DISPCOR using the comparison spectra.

We had two, three, and five exposures of approximately $\lambda \lambda 3830-4485$ for $\kappa$ Cnc, HR 7245 , and $\xi$ Oct, respectively. Further for both $\kappa$ Cnc and HR 7245, we had two and three exposures, respectively, covering approximately $\lambda \lambda 4500-5120$. For $\kappa$ Cnc we also had two exposures covering $\lambda \lambda 4090-4800$. Within these spectral ranges not all of the spectra were equally useful. We obtained the spectra for $\xi$ Oct in November 1994 and for $\kappa$ Cnc and HR 7245 in April 1995. The signal-to-noise ratio $(S / N)$ of these spectra is typically of at least order 300 in the centers of well exposed orders.

The REOSC spectrograms are better focused at the centers of the orders. There are also wavelength errors

\footnotetext{
${ }^{1}$ IRAF is distributed by the National Optical Astronomical Observatories which is operated by the Association of Universities for Research in Astronomy, Inc., under a cooperative agreement with the National Science Foundation.
}

which we believe the IRAF reduction produces (as one of us SJA has had similar problems reducing KPNO coudé spectrograms). One can correct the wavelength scale of a particular order by using a second-order correction in the difference between the observed and laboratory wavelengths after sufficient lines have been identified. But then all radial velocity information is lost.

After the individual orders were extracted, they were normalized with REDUCE (Hill \& Fisher 1986). The spectra of each star were coadded order by order to reduce the noise. One spectrum for each star was selected as a standard. Then its orders were cross-correlated with those of the other spectra using VCROSS before the orders were added using TSTACK. Finally the spectra were measured using VLINE. When broad lines such as those of He I occur near the peak of an order uncertainties in the placement of the continuum arise.

The stellar lines were identified with the general references A Multiplet Table of Astrophysical Interest (Moore 1945) and Wavelengths and Transition Probabilities for Atoms and Atomic Ions, Part 1 (Reader \& Corliss 1980) as well as the more specialized references P II (Svendenius et al. 1983), P III (Magnusson \& Zetterberg 1987), Mn II (Iglesias \& Velasco 1964), Fe II (Johansson 1978), and Ga II (Isberg \& Litzen 1985).

$\xi$ Oct is a weak-lined star with relatively few lines per order at the observed spectral resolution. The unpublished line list of Adelman et al. (1993) based on Anglo-Australian Telescope (AAT) echelle spectrograph data was a useful guide in the regions of spectral overlap. We performed a least squares regression for 24 lines with equivalent widths less than $130 \mathrm{~m} \AA$ and found:

$$
W_{\lambda}(\mathrm{CASLEO})=-1.717+1.203 W_{\lambda}(\mathrm{AAT})
$$

which reflects the lower resolution and $S / N$ of the CASLEO data. The agreement of the two data sets is 
Table 2. The analysis of the metal lines from CASLEO Spectra for $\xi$ Oct

\begin{tabular}{|c|c|c|c|c|c|}
\hline mult. & $\lambda(\AA)$ & $\log g f$ & Ref. & $W_{\lambda}(\mathrm{mA})$ & $\log N / N_{T}$ \\
\hline C II & & & \multicolumn{3}{|c|}{$\left(\log C / N_{T}=-3.63 \pm 0.32\right)$} \\
\hline \multirow[t]{2}{*}{4} & 3918.98 & -0.54 & WS & 26 & -3.84 \\
\hline & 3920.69 & -0.24 & WS & 44 & -3.79 \\
\hline 6 & 4267.15 & +0.97 & WS & 111 & -3.26 \\
\hline \multicolumn{3}{|c|}{ Mg II } & \multicolumn{3}{|c|}{$\left(\log \mathrm{Mg} / N_{T}=-4.71 \pm 0.17\right)$} \\
\hline 4 & 4481.23 & +0.97 & WM & 275 & -4.46 \\
\hline 9 & 4433.99 & -0.90 & WS & 15 & -4.78 \\
\hline \multirow[t]{2}{*}{10} & 4384.64 & -0.78 & WS & 19 & -4.81 \\
\hline & 4390.58 & -0.53 & WS & 27 & -4.81 \\
\hline \multicolumn{3}{|l|}{ Si II } & \multicolumn{3}{|c|}{$\left(\log \mathrm{Si} / N_{T}=-4.48 \pm 0.10\right)$} \\
\hline 1 & 3853.66 & -1.44 & LA & 68 & -4.59 \\
\hline & 3856.02 & -0.49 & LA & 120 & -4.43 \\
\hline & 3862.05 & -0.74 & LA & 112 & -4.34 \\
\hline 3 & 4128.07 & +0.38 & LA & 105 & -4.51 \\
\hline & 4130.89 & +0.53 & LA & 116 & -4.51 \\
\hline S II & & & \multicolumn{3}{|c|}{$\left(\log \mathrm{S} / N_{T}=-4.89 \pm 0.00\right)$} \\
\hline \multirow[t]{2}{*}{49} & 4282.63 & -0.01 & WS & 7 & -4.88 \\
\hline & 4294.43 & +0.56 & WS & 16 & -4.89 \\
\hline \multicolumn{3}{|l|}{$\mathrm{Ca}$ II } & \multicolumn{3}{|c|}{$\left(\log \mathrm{Ca} / N_{T}=-5.56\right)$} \\
\hline 1 & 3933.66 & +0.14 & \multirow[t]{2}{*}{ WM } & 129 & -5.56 \\
\hline \multicolumn{3}{|l|}{ Cr II } & & \multicolumn{2}{|c|}{$\left(\log \mathrm{Cr} / N_{T}=-6.39\right)$} \\
\hline 31 & 4242.38 & -1.33 & KX & 6 & -6.39 \\
\hline \multicolumn{3}{|l|}{ Fe II } & \multicolumn{3}{|c|}{$\left(\log \mathrm{Fe} / N_{T}=-4.80 \pm 0.20\right)$} \\
\hline \multirow[t]{5}{*}{27} & 4233.16 & -2.00 & MF & 46 & -4.66 \\
\hline & 4273.32 & -3.34 & $\mathrm{MF}$ & 4 & -4.91 \\
\hline & 4303.17 & -2.49 & $\mathrm{MF}$ & 14 & -5.12 \\
\hline & 4351.76 & -2.10 & MF & 26 & -5.12 \\
\hline & 4416.82 & -2.60 & MF & 24 & -4.64 \\
\hline \multirow[t]{2}{*}{28} & 4178.86 & -2.48 & MF & 26 & -4.78 \\
\hline & 4296.57 & -3.01 & $\mathrm{MF}$ & 9 & -4.86 \\
\hline 173 & 3906.04 & -1.83 & MF & 14 & -4.56 \\
\hline $\mathrm{J}$ & 4357.58 & -2.10 & $\mathrm{KX}$ & 5 & -4.59 \\
\hline
\end{tabular}

\footnotetext{
Ref: KX = Kurucz (1995)

LA = Lanz \& Artru (1985)

MF = Martin et al. (1988) and Fuhr et al. (1988)

$\mathrm{WM}=$ Wiese \& Martin (1980)

WS $=$ Wiese et al. $(1966,1969)$
}

much better longward of $4150 \AA$ than shortward of this value.

For HR 7245, a linear regression analysis with 39 equivalent widths from Guthrie (1984) who measured a $2.4 \AA \mathrm{mm}^{-1} \mathrm{DAO}$ IIaO spectrogram yielded

$$
W_{\lambda}(\mathrm{CASLEO})=5.212+0.617 W_{\lambda}(\mathrm{DAO})
$$

In addition we compared our values with 25 lines from Zakharova (1994) who used four $9 \AA \mathrm{mm}^{-1}$ spectrograms and found

$$
W_{\lambda}(\mathrm{CASLEO})=-6.934+0.775 W_{\lambda}(\mathrm{Z})
$$

But for $\kappa$ Cnc, a comparison with 26 lines in the $\lambda \lambda 4500-4640$ region with Adelman's (1987) study which
Table 3. Comparison of Abundances for $\xi$ Oct

\begin{tabular}{rcrcc}
\hline Species & AAT & \multicolumn{3}{c}{ CASLEO } \\
& $\log N / H$ & $n$ & $\log N / H$ & $n$ \\
\hline He I & -1.14 & 3 & -1.11 & 9 \\
C II & -3.79 & 3 & -3.59 & 3 \\
O I & -3.24 & 1 & $\ldots$ & $\ldots$ \\
O II & -3.42 & 5 & $\ldots$ & $\ldots$ \\
Mg II & -4.68 & 2 & -4.67 & 4 \\
Si II & -4.73 & 5 & -4.44 & 5 \\
S II & -5.02 & 14 & -4.85 & 2 \\
Ca II & -6.08 & 1 & -5.52 & 1 \\
Ti II & -7.14 & 1 & $\ldots$ & $\ldots$ \\
Cr II & -6.49 & 1 & -6.35 & 1 \\
Fe I & -4.27 & 3 & $\ldots$ & $\ldots$ \\
Fe II & -4.72 & 20 & -4.76 & 9 \\
Ni II & -5.91 & 3 & $\ldots$ & $\ldots$ \\
\hline
\end{tabular}

used a coaddition of $102.4 \AA \mathrm{mm}^{-1}$ DAO IIaO spectrograms yielded

$$
W_{\lambda}(\mathrm{CASLEO})=-2.935+1.280 W_{\lambda}(\mathrm{DAO})
$$

which is a result similar to that found for $\xi$ Oct. For $\kappa$ Cnc we had a complete line identification list which we lacked for HR 7245 and could avoid blended lines. It is very difficult to reconcile these results which indicate the CASLEO equivalent widths are both too big and too small. We attempted to avoid blended lines in these comparisons.

\section{Stellar parameters}

We used Kurucz (1995)'s ATLAS9 model atmospheres which are the most appropriate for B and A type main sequence band stars. Programs SYNSPEC (Hubeny et al. 1994) and WIDTH9 (Kurucz, private communication) were employed, respectively, to determine the helium and metal abundances. The adopted metal-line damping constants were the default semi-classical approximations, except for iron-peak element lines, whose values were based on the data of Kurucz (1995); for C II, multiplet $6, \mathrm{Mg}$ II multiplet 4, and Ca II multiplet 1, where the adopted values for the Stark broadening used the data of SahalBréchot (1969); and for Si II multiplets 1 and 3, the damping constants were from Lanz et al. (1988). We used a $4 \%$ scattered light correction to account for light scattered along the direction of the dispersion, which is an appropriate value for clean optical systems.

To obtain initial estimates of the effective temperatures and surface gravities, we used homogeneous Stromgren uvby $\beta$ photometry (Hauck \& Mermilliod 1990) and the formulation of Napiwotzki et al. (1993): $T_{\text {eff }}=$ $13660 \mathrm{~K}, \log g=3.79$ for $\kappa \mathrm{Cnc}, T_{\text {eff }}=12360 \mathrm{~K}, \log$ $g=3.45$ for HR 7245, and $T_{\text {eff }}=14130 \mathrm{~K}, \log g=3.93$ for $\xi$ Oct. The photometry indicates that HR 7245 may be 
Table 4. The analysis of the metal lines from CASLEO Spectra for $\kappa$ Cnc

\begin{tabular}{|c|c|c|c|c|c|}
\hline mult. & $\lambda(\AA)$ & $\log g f$ & Ref. & $W_{\lambda}(\mathrm{m} \check{A})$ & $\log N / N_{T}$ \\
\hline P II & & & \multicolumn{3}{|c|}{$\left(\log \mathrm{P} / N_{T}=-4.59 \pm 0.19\right)$} \\
\hline \multirow[t]{4}{*}{13} & 4927.20 & -0.68 & WS & 10 & -4.49 \\
\hline & 4943.50 & +0.06 & WS & 29 & -4.41 \\
\hline & 4954.39 & -0.54 & WS & 14 & -4.43 \\
\hline & 4969.70 & -0.19 & WS & 23 & -4.39 \\
\hline 15 & 4658.31 & -0.31 & WS & 11 & -4.86 \\
\hline 28 & 4679.03 & -0.40 & KX & 9 & -4.75 \\
\hline $\mathrm{S}$ & 4935.63 & -0.16 & WS & 12 & -4.51 \\
\hline \multicolumn{3}{|l|}{ Cr II } & \multicolumn{3}{|c|}{$\left(\log \mathrm{Cr} / N_{T}=-6.17 \pm 0.11\right)$} \\
\hline \multirow[t]{3}{*}{30} & 4824.12 & -1.22 & MF & 24 & -6.02 \\
\hline & 4848.24 & -1.14 & $\mathrm{MF}$ & 21 & -6.20 \\
\hline & 4876.41 & -1.46 & $\mathrm{MF}$ & 10 & -6.31 \\
\hline 190 & 4912.46 & -0.95 & $\mathrm{KX}$ & 3 & -6.12 \\
\hline \multicolumn{3}{|c|}{ Mn II } & \multicolumn{3}{|c|}{$\left(\log \mathrm{Mn} / N_{T}=-4.43 \pm 0.21\right)$} \\
\hline \multirow[t]{19}{*}{-} & 4717.26 & -1.86 & $\mathrm{KX}$ & 18 & -4.40 \\
\hline & 4727.84 & -2.02 & KX & 35 & -4.44 \\
\hline & 4730.40 & -2.15 & KX & 34 & -4.15 \\
\hline & 4738.30 & -2.24 & KX & 20 & -4.49 \\
\hline & 4749.11 & -2.00 & KX & 14 & -4.57 \\
\hline & 4755.73 & -1.24 & KX & 50 & -4.51 \\
\hline & 4764.73 & -1.35 & KX & 33 & -4.94 \\
\hline & 4791.78 & -1.72 & KX & 28 & -4.37 \\
\hline & 4806.82 & -1.56 & KX & 44 & -4.37 \\
\hline & 4811.62 & -2.34 & KX & 17 & -4.46 \\
\hline & 4830.06 & -1.85 & KX & 24 & -4.38 \\
\hline & 4839.74 & -1.86 & KX & 17 & -4.61 \\
\hline & 4842.32 & -2.00 & KX & 22 & -4.29 \\
\hline & 4847.61 & -1.81 & KX & 20 & -4.88 \\
\hline & 4851.54 & -2.61 & KX & 13 & -4.36 \\
\hline & 4920.44 & -2.09 & KX & 25 & -4.43 \\
\hline & 4921.23 & -1.58 & KX & 33 & -4.15 \\
\hline & 5102.52 & -1.93 & KX & 30 & -4.17 \\
\hline & 5107.09 & -1.48 & KX & 19 & -4.34 \\
\hline Fe II & & & \multicolumn{3}{|c|}{$\left(\log \mathrm{Fe} / N_{T}=-4.20 \pm 0.20\right)$} \\
\hline 36 & 4993.35 & -3.65 & $\mathrm{MF}$ & 16 & -4.13 \\
\hline 37 & 4663.70 & -4.28 & KX & 11 & -3.68 \\
\hline \multirow[t]{2}{*}{42} & 4923.93 & -1.32 & $\mathrm{MF}$ & 77 & -4.36 \\
\hline & 5018.45 & -1.22 & $\mathrm{MF}$ & 93 & -3.97 \\
\hline \multirow[t]{2}{*}{43} & 4656.87 & -3.63 & $\mathrm{MF}$ & 8 & -4.49 \\
\hline & 4731.44 & -3.36 & $\mathrm{MF}$ & 19 & -4.26 \\
\hline \multirow[t]{18}{*}{-} & 4820.83 & -0.69 & $\mathrm{KX}$ & 7 & -4.19 \\
\hline & 4826.68 & -0.44 & $\mathrm{KX}$ & 5 & -4.57 \\
\hline & 4908.15 & -0.30 & $\mathrm{KX}$ & 10 & -4.33 \\
\hline & 4913.29 & +0.01 & KX & 12 & -4.56 \\
\hline & 4948.10 & -0.32 & $\mathrm{KX}$ & 7 & -4.52 \\
\hline & 4948.79 & -0.01 & $\mathrm{KX}$ & 15 & -4.38 \\
\hline & 4951.58 & +0.18 & KX & 16 & -4.54 \\
\hline & 4977.03 & +0.04 & KX & 18 & -4.32 \\
\hline & 4984.48 & +0.01 & $\mathrm{KX}$ & 20 & -4.22 \\
\hline & 4990.51 & +0.18 & $\mathrm{KX}$ & 24 & -4.24 \\
\hline & 5001.96 & +0.90 & $\mathrm{KX}$ & 40 & -4.44 \\
\hline & 5004.20 & +0.50 & KX & 29 & -4.41 \\
\hline & 5006.80 & -0.43 & KX & 8 & -4.33 \\
\hline & 5007.74 & -0.20 & $\mathrm{KX}$ & 15 & -4.19 \\
\hline & 5009.02 & -0.42 & KX & 7 & -4.37 \\
\hline & 5021.59 & -0.30 & $\mathrm{KX}$ & 11 & -4.27 \\
\hline & 5026.81 & -0.22 & KX & 18 & -4.04 \\
\hline & 5030.63 & +0.40 & $\mathrm{KX}$ & 22 & -4.54 \\
\hline
\end{tabular}

Table 4. continued

\begin{tabular}{ccccrc}
\hline mult. & $\lambda(\AA)$ & $\log g f$ & Ref. & $W_{\lambda}(\mathrm{m} \AA)$ & $\log N / N_{T}$ \\
\hline Fe II (cont.) & & & & \\
$-\quad 5031.89$ & -0.78 & KX & 6 & -4.09 \\
5032.71 & +0.11 & KX & 26 & -4.05 \\
5033.96 & -0.73 & KX & 9 & -3.97 \\
5035.71 & +0.61 & KX & 31 & -4.44 \\
5045.11 & -0.13 & KX & 35 & -3.54 \\
5047.64 & -0.07 & KX & 20 & -4.12 \\
5060.26 & -0.52 & KX & 7 & -4.28 \\
5061.72 & +0.22 & KX & 20 & -4.43 \\
5067.89 & -0.20 & KX & 17 & -4.09 \\
5070.90 & +0.24 & KX & 27 & -4.20 \\
5074.05 & -1.97 & KX & 13 & -4.08 \\
5075.76 & +0.28 & KX & 24 & -4.26 \\
5082.23 & -0.10 & KX & 20 & -4.04 \\
5086.31 & -0.48 & KX & 14 & -3.89 \\
5087.30 & -0.50 & KX & 12 & -4.01 \\
5093.58 & +0.11 & KX & 27 & -4.03 \\
5097.27 & +0.31 & KX & 39 & -3.82 \\
5106.11 & -0.19 & KX & 28 & -3.71 \\
\hline
\end{tabular}

For $g f$ value references: see Table 2

Table 5. Comparison DAO and CASLEO Abundances for $\kappa$ Cnc $(\log N / H)$

\begin{tabular}{lcrcr}
\hline Species & DAO & $n$ & CASLEO & $n$ \\
\hline P II & $-4.61 \pm 0.15$ & 13 & $-4.56 \pm 0.19$ & 7 \\
Cr II & $-6.37 \pm 0.16$ & 22 & $-6.14 \pm 0.11$ & 4 \\
Mn II & $-4.39 \pm 0.22$ & 87 & $-4.40 \pm 0.21$ & 19 \\
Fe II & $-4.47 \pm 0.25$ & 101 & $-4.17 \pm 0.25$ & 42 \\
\hline
\end{tabular}

reddened. By comparing spectrophotometric fluxes (Adelman \& Pyper 1979) and the $\mathrm{H} \gamma$ profile (Adelman 1987) for $\kappa$ Cnc with the predictions of a solar composition ATLAS9 model, we found $T_{\text {eff }}=13000 \mathrm{~K}, \log g=3.65$ while for a $[+0.2]$ dex model these values become $T_{\text {eff }}=12920$ $\mathrm{K}, \log g=3.75$. For $\kappa$ Cnc, Adelman (1987) used $T_{\text {eff }}=$ $13125 \mathrm{~K}, \log g=3.45$ and found a mean metallicity close to solar. Thus we chose the solar composition model. For HR 7245, Guthrie (1984) adopted $T_{\text {eff }}=12300 \mathrm{~K}, \log g=$ 3.8 and Roby \& Lambert (1990) $T_{\text {eff }}=12350 \mathrm{~K}, \log g=$ 3.6 , in better temperature agreement than surface gravity agreement with our photometric values. For $\xi$ Oct, Adelman et al. (1993) used $T_{\text {eff }}=13625 \mathrm{~K}, \log g=4.0$ while Hardorp et al. (1986) $T_{\text {eff }}=14000 \mathrm{~K}, \log g=4.0$. Such differences are within the uncertainties of $\pm 500 \mathrm{~K}$ in effective temperature and \pm 0.2 dex in log surface gravity.

For $\xi$ Oct we adopted a microturbulence of $0.0 \mathrm{~km}$ $\mathrm{s}^{-1}$ as we did not have a sufficient number of lines for a proper analysis and as recent analyses of other superficially normal middle B stars found this value (Adelman 
Table 7. Comparison of Abundances for HR7245 $(\log N / H)$

\begin{tabular}{lrrrrrrr}
\hline Species & Guthrie & $n$ & Zakharova & $n$ & This Paper & $n$ & Sun \\
\hline He I & $\ldots$ & $\ldots$ & -1.47 & 2 & -1.68 & 5 & -1.00 \\
C II & $\ldots$ & $\ldots$ & -3.50 & 2 & -3.95 & 2 & -3.43 \\
O I & $\ldots$ & $\ldots$ & $\ldots$ & $\ldots$ & -3.15 & 1 & -3.14 \\
Mg II & -5.1 & 2 & $\ldots$ & $\ldots$ & -4.71 & 2 & -4.42 \\
Si II & $\ldots$ & $\ldots$ & -4.02 & 4 & -4.31 & 3 & -4.45 \\
P II & -5.2 & 7 & $\ldots$ & $\ldots$ & -5.51 & 3 & -6.55 \\
S II & $\ldots$ & $\ldots$ & $\ldots$ & $\ldots$ & -5.41 & 4 & -4.67 \\
Ca II & $\ldots$ & $\ldots$ & $\ldots$ & $\ldots$ & -4.80 & 1 & -5.64 \\
Sc II & -8.2 & 2 & $\ldots$ & $\ldots$ & -8.69 & 1 & -8.90 \\
Ti II & -6.5 & 18 & -6.15 & 10 & -6.34 & 22 & -7.01 \\
V II & $>-8.1$ & 2 & -6.46 & 3 & $\ldots$ & $\ldots$ & -8.00 \\
Cr II & -5.9 & 9 & -5.80 & 5 & -5.84 & 31 & -6.26 \\
Mn I & $\ldots$ & $\ldots$ & $\ldots$ & $\ldots$ & -4.30 & 1 & -6.45 \\
Mn II & -5.4 & 20 & -4.62 & 36 & -4.84 & 57 & -6.45 \\
Fe I & $\ldots$ & $\ldots$ & -4.48 & 1 & -4.43 & 5 & -4.52 \\
Fe II & -4.6 & 9 & -4.29 & 28 & -4.54 & 78 & -4.52 \\
Fe III & $\ldots$ & $\ldots$ & -4.02 & 1 & -4.85 & 1 & -4.52 \\
Ni II & $>-6.5$ & 3 & $\ldots$ & $\ldots$ & -6.45 & 1 & -5.75 \\
Ga II & -5.6 & 3 & -5.48 & 2 & -5.93 & 2 & -9.11 \\
Sr II & $\ldots$ & $\ldots$ & -8.33 & 2 & -8.73 & 2 & -9.10 \\
Y II & -8.6 & 2 & -7.60 & 1 & -8.12 & 1 & -9.76 \\
Zr II & $>-8.8$ & 1 & -6.86 & 2 & -7.71 & 4 & -9.40 \\
Xe II & $\ldots$ & $\ldots$ & -4.49 & 5 & $\ldots$ & $\ldots$ & $\ldots$ \\
Ce II & $\ldots$ & $\ldots$ & $\ldots$ & $\ldots$ & -6.72 & 1 & -10.45 \\
Pt II & $>-6.9$ & 2 & $\ldots$ & $\ldots$ & $\ldots$ & $\ldots$ & -10.20 \\
Hg II & $\ldots$ & $\ldots$ & $\ldots$ & $\ldots$ & -6.52 & 1 & $\ldots$ \\
\hline
\end{tabular}

1994a). For $\kappa$ Cnc we used $0.0 \mathrm{~km} \mathrm{~s}^{-1}$ as found by Adelman (1987). When we tried find the microturbulence from the Fe II lines of HR 7245, they indicated a value of a few $\mathrm{km} \mathrm{s}^{-1}$ which is contrary to Adelman's (1994b) result that most HgMn stars have little or no microturbulence. Our result reflects small errors in the $g f$-values and equivalent widths and that almost all the Fe II lines are on the linear part of the curve-of-growth. Thus we adopted a value of $0.0 \mathrm{~km} \mathrm{~s}^{-1}$ for this abundance analysis.

\section{The elemental abundance analyses}

For $\xi$ Oct the derived $\mathrm{He} / \mathrm{H}$ values (Table 1) are slightly smaller than 0.085 found by Adelman et al. (1993) using only $\lambda 4026$ and $\lambda 4388$. The CASLEO values show a greater scatter than those from the AAT. We did not analyze any He I lines for $\kappa$ Cnc as we did not think that we could improve the value from DAO spectrograms. For HR 7245 we analyzed 5 lines and found $\mathrm{He} / \mathrm{H}=0.02$, which is a value appropriate to a $\mathrm{HgMn}$ star.

We analyzed $\xi$ Oct using both CASLEO and AAT data. Table 2 contains the metal line results from our spectra. For each line, it contains the multiplet number, the wavelength in $\AA$, the equivalent width in $\mathrm{m} \AA$, the $g f$ value and its source, and the derived abundance $\left(\log N / N_{T}\right)$ where $N_{T}$ is the total number of atoms per unit volume. Table 3 summarizes the abundance results. The CASLEO data contains a few lines which the AAT data lacks. The AAT results are given only in summary form as the details are in Adelman et al. (1993). In general $\xi$ Oct now appears to be slightly more metal rich. The difference between the abundance of iron as derived from the Fe I and Fe II lines has been increased, but this may represent nonLTE effects. The differences are usually larger when only strong lines are involved in the analyses, e.g. Si II and Ca II. Those for species which have only weak lines are about 0.10 dex different.

For $\kappa$ Cnc, we analyzed lines only in the $\lambda \lambda 4640-5110$ region (Table 4) as these lines supplement those found using DAO spectrograms and as the DAO data is of higher spectroscopic quality in resolution if not in $S / N$. For comparison we reanalyzed the lines in Adelman (1987) for those atomic species in common (Table 5). The CASLEO values on average make $\kappa$ Cnc about $0.14 \pm 0.15$ dex more metal rich, which is about that expected based on the difference in equivalent width scales. This suggests that this difference was reasonably determined well for this star although part of the discrepancies may be due to small $g f$ value systematics. 
Table 6 contains the analysis of the metal lines for HR 7245 while Table 7 compares our results with those of Guthrie (1984) and Zakharov (1994). Our values are often between those of these investigators which is somewhat surprising given the equivalent width comparisons. Compared to other HgMn stars of similar temperature (Adelman 1994b), its abundances fall mostly within the run of values. It apparently has the most nearly solar $\mathrm{O}$ abundance and its Ca abundance is the most above solar.

\section{Final comments}

Since we took our spectrograms, the CASLEO staff has made a substantial effort to improve the quality of the spectrograms taken with the REOSC echelle. This should alleviate some of the problems which we discussed. A further critical task is to better determine the relation of the CASLEO equivalent width scale to that of other major spectroscopic instruments.

Still we are working with an instrument which does not have the resolution of the most powerful coudé and echelle spectrographs in the blue. But in the yellow and the red, there are fewer lines and blending is less severe. The spectra of the HgMn stars in $\lambda \lambda 4640-5100$ show many unidentified lines along with a nice selection of He I, Si II, P II, Ti II, Cr II, Mn II, and Fe II lines plus those of more exotic species such as Xe II. Kurucz (1995) has calculated the $g f$ values of most of these lines. Part of the spectral region between $\lambda 5100$ and $\lambda 10000$ may prove even more interesting and provide abundances of elements such as $\mathrm{C}, \mathrm{N}$, and $\mathrm{O}$, which often have not been obtained in the photographic region. Further cataloging the wavelengths and strengths of unidentified lines and then performing correlations with strengths of lines of known species may provide useful information to atomic spectroscopists who are extending analyses of astrophysically common and rare species.

Acknowledgements. OIP is grateful to the staff of CASLEO for their assistance during the observing runs, especially A. De Franceschi, the night assistant. She also thanks J.L. Guiliani, H. Carbonari, and Dr. A. Gulliver for help in installing computer codes from Dr. R.L. Kurucz. She appreciates useful discussions concerning spectral reductions with Dr. H. Levato, Dr. B. Garcia, and L. Navarro. The authors acknowledge used of the CCD and data reduction acquisition system supported by US NSF Grant AST 90-15827 to R.M. Rich. SJA thanks The Citadel Development Foundation for several grants which supported in part this collaboration.

\section{References}

Adelman S.J., 1987, MNRAS 228, 573
Adelman S.J., 1994a, MNRAS 271, 355

Adelman S.J., 1994b, MNRAS 266, 97

Adelman S.J., Pyper D.M., 1979, AJ 84, 1603

Adelman S.J., Richardson R.D., Wahlgren G.M., 1993, PASP 105, 327

Biemont E., Grevesse N., Hannaford P., Lowe R.M., 1981, ApJ 248,867

Dworestsky M.M., 1980, A\&A 84, 350

Fuhr J.R., Martin G.A., Wiese, W.L., 1988, J. Phys. Chem. Ref. Data 15, Suppl. 4

Grevesse N., Biemont E., Hannaford P., Lowe R.M., 1981, Upper Main Sequence Stars, 23rd Liege Astrophys. Coll., p. 211

Guthrie B.N.G., 1984, MNRAS 206, 65

Guthrie B.N.G., 1985, MNRAS 216, 1

Hannaford P., Lowe R.M., Grevesse N., Biemont E., 1982, ApJ 261,736

Hardorp J., Cugier H., Koratjar A., Scott J., 1986 In: New Insights in Astrophysics, ESA SP-263, Paris, p. 377

Hauck B., Mermilliod M., 1990, A\&AS 86, 107

Hill G., Fisher W.A., 1986, Publ. Dom. Astrophys. Obs. Victoria 16, 159

Hoffleit D., 1982, The Bright Star Catalogue, 4th edition, Yale University Observatory, New Haven

Hubeny I., Lanz T., Jeffery C.S., 1994, Daresbury Lab. New. Anal. Astron. Spectra 20, 30

Iglesias L., Velasco R., 1964, Publ. Inst. Opt. Madrid, No. 23

Isberg B., Litzen U., 1985, Phys. Scripta 31, 533

Johansson S., 1978, Phys. Scripta 18, 217

Jugaku J., Sargent W.L.W., Greenstein J.L., 1961, ApJ 134, 783

Kurucz R.L., 1995, ASP Conf. Ser. San Francisco 78, 205

Lanz T., Artru M.-C., 1985, Phys. Scripta 32, 155

Lanz T., Dimitrijevic, M.S., Artru, M.-C., 1988, A\&A 192, 249

Magnusson C.E., Zetterberg P.O., 1977, Phys. Scripta 15, 237

Martin G.A., Fuhr J.R., Wiese W.L., 1988, J. Phys. Chem. Ref. Data 15, Suppl. 3

Moore C.E., 1945, A Multiple Table of Astrophysical Interest Princeton University Observatory, Princeton

Napiwotzki R., Schönberner D., Wenske V., 1993, A\&A 268, 653

Reader J., Corliss C.H., 1980, NSRDS-NBS 68, Part 1, US Government Printing Office, Washington, DC

Roby S.W., Lambert S.W., 1990, ApJS 73, 67

Sahal-Bréchot S., 1969, A\&A 2, 322

Svendenius N., Magnusson C.E., Zetterberg P.O., 1983, Phys. Scripta 27, 339

Wiese W.L., Martin G.A., 1980, NSRDS-NBS 68 Part 2, US Government Printing Office, Washington, DC

Wiese W.L., Smith M.W., Glennon B.M., 1966, NSRDS-NBS 4, US Government Printing, Office, Washington, DC

Wiese W.L., Smith M.W., Miles B.M., 1969, NSRDS-NBS 22, US Government Printing Office, Washington, DC

Zakharova L.A., 1994, Astron. Zh. 71, 588 
Table 6. The analysis of the metal lines HR 7245

\begin{tabular}{|c|c|c|c|c|c|}
\hline mult. & $\lambda(\AA)$ & $\log g f$ & Ref. & $W_{\lambda}(\mathrm{m} \AA)$ & $\log N / N_{T}$ \\
\hline C II & & & \multicolumn{3}{|c|}{$\left(\log \mathrm{C} / N_{T}=-3.96 \pm 0.30\right)$} \\
\hline 4 & 3918.98 & -0.54 & WS & 13 & -4.17 \\
\hline 6 & 4267.25 & +0.97 & WS & 44 & -3.75 \\
\hline O I & & & & \multicolumn{2}{|c|}{$\left(\log \mathrm{O} / N_{T}=-3.16\right)$} \\
\hline 5 & 4368.30 & -1.77 & WS & 13 & -3.16 \\
\hline $\mathrm{Mg}$ II & & & \multicolumn{3}{|c|}{$\left(\log \mathrm{Mg} / N_{T}=-4.72 \pm 0.36\right)$} \\
\hline 4 & 4481.23 & +0.97 & WM & 263 & -4.47 \\
\hline 9 & 4428.00 & -1.20 & WS & 8 & -4.98 \\
\hline Si II & & & \multicolumn{3}{|c|}{$\left.\log \mathrm{Si} / N_{T}=-4.32 \pm 0.14\right)$} \\
\hline 1 & 3856.02 & -0.49 & LA & 126 & -4.40 \\
\hline 3 & 4128.07 & +0.38 & LA & 128 & -4.16 \\
\hline & 4130.89 & +0.53 & LA & 121 & -4.39 \\
\hline P II & & & \multicolumn{3}{|c|}{$\left(\log \mathrm{P} / N_{T}=-5.52 \pm 0.28\right)$} \\
\hline 13 & 4943.50 & +0.06 & WS & 5 & -5.51 \\
\hline 15 & 4602.08 & +0.74 & WM & 27 & -5.18 \\
\hline- & 4420.71 & -0.34 & WS & 7 & -5.86 \\
\hline S II & & & \multicolumn{3}{|c|}{$\left(\log \mathrm{S} / N_{T}=-5.42 \pm 0.30\right)$} \\
\hline 7 & 5032.41 & +0.18 & WS & 15 & -4.98 \\
\hline 44 & 4145.10 & +0.44 & WS & 5 & -5.49 \\
\hline & 4153.10 & +0.62 & WS & 6 & -5.58 \\
\hline & 4162.70 & +0.78 & WS & 6 & -5.64 \\
\hline $\mathrm{Ca} \mathrm{II}$ & & & & \multicolumn{2}{|c|}{$\left(\log \mathrm{Ca} / N_{T}=-4.81\right)$} \\
\hline 1 & 3933.66 & +0.13 & WM & 312 & -4.81 \\
\hline Sc II & & & & \multicolumn{2}{|c|}{$\left(\log \mathrm{Sc} / N_{T}=-8.70\right)$} \\
\hline 7 & 4246.83 & +0.32 & $\mathrm{MF}$ & 14 & -8.70 \\
\hline Ti II & & & \multicolumn{3}{|c|}{$\left(\log \mathrm{Ti} / N_{T}=-6.35 \pm 0.31\right)$} \\
\hline \multirow[t]{2}{*}{19} & 4443.80 & -0.70 & $\mathrm{MF}$ & 30 & -6.74 \\
\hline & 4450.49 & -1.45 & $\mathrm{MF}$ & 10 & -6.67 \\
\hline \multirow[t]{2}{*}{20} & 4287.89 & -2.02 & MF & 11 & -6.06 \\
\hline & 4294.09 & -1.11 & $\mathrm{MF}$ & 16 & -6.76 \\
\hline \multirow[t]{2}{*}{34} & 3900.56 & -0.45 & $\mathrm{MF}$ & 40 & -6.67 \\
\hline & 3913.46 & -0.53 & $\mathrm{MF}$ & 46 & -6.44 \\
\hline \multirow[t]{2}{*}{40} & 4290.22 & -1.12 & $\mathrm{MF}$ & 16 & -6.72 \\
\hline & 4417.72 & -1.43 & $\mathrm{MF}$ & 31 & -5.95 \\
\hline \multirow[t]{5}{*}{41} & 4300.05 & -0.77 & $\mathrm{MF}$ & 45 & -6.21 \\
\hline & 4301.93 & -1.16 & $\mathrm{MF}$ & 11 & -6.89 \\
\hline & 4312.86 & -1.16 & $\mathrm{MF}$ & 28 & -6.30 \\
\hline & 4314.98 & -1.13 & $\mathrm{MF}$ & 27 & -6.36 \\
\hline & 4320.96 & -1.87 & MF & 12 & -6.11 \\
\hline 50 & 4563.76 & -0.96 & $\mathrm{MF}$ & 37 & -6.24 \\
\hline 82 & 4571.97 & -0.53 & $\mathrm{MF}$ & 39 & -6.43 \\
\hline 92 & 4805.09 & -1.10 & $\mathrm{MF}$ & 16 & -6.28 \\
\hline \multirow[t]{2}{*}{104} & 4367.65 & -1.27 & $\mathrm{MF}$ & 13 & -5.98 \\
\hline & 4386.86 & -1.26 & MF & 8 & -6.29 \\
\hline 105 & 4163.64 & -0.40 & $\mathrm{MF}$ & 33 & -6.24 \\
\hline 114 & 4911.18 & -0.34 & $\mathrm{MF}$ & 14 & -6.63 \\
\hline \multirow[t]{2}{*}{115} & 4456.63 & -1.66 & KX & 5 & -5.84 \\
\hline & 4411.08 & -1.06 & $\mathrm{MF}$ & 14 & -5.93 \\
\hline Cr II & & & \multicolumn{3}{|c|}{$\left(\log \mathrm{Cr} / N_{T}=-5.85 \pm 0.27\right)$} \\
\hline 18 & 4172.60 & -2.36 & KX & 6 & -6.24 \\
\hline 19 & 4051.97 & -2.19 & KX & 23 & -5.66 \\
\hline 20 & 3979.51 & -0.73 & KX & 16 & -6.11 \\
\hline 26 & 4179.43 & -1.77 & $\mathrm{KX}$ & 14 & -6.04 \\
\hline 30 & 4812.34 & -1.80 & MF & 11 & -6.14 \\
\hline & 4824.12 & -1.22 & MF & 47 & -5.53 \\
\hline & 4836.22 & -2.25 & MF & 10 & -5.72 \\
\hline & 4848.24 & -1.14 & $\mathrm{MF}$ & 32 & -6.06 \\
\hline & 4876.41 & -1.46 & $\mathrm{MF}$ & 23 & -6.01 \\
\hline
\end{tabular}

Table 6. continued

\begin{tabular}{|c|c|c|c|c|c|}
\hline mult. & $\lambda(\AA)$ & $\log g f$ & Ref. & $W_{\lambda}(\mathrm{m} \AA)$ & $\log N / N_{T}$ \\
\hline \multicolumn{6}{|c|}{ Cr II (cont.) } \\
\hline \multirow[t]{4}{*}{31} & 4261.92 & -1.53 & KX & 35 & -5.61 \\
\hline & 4275.57 & -1.71 & $\mathrm{KX}$ & 25 & -5.71 \\
\hline & 4252.62 & -2.02 & KX & 19 & -5.59 \\
\hline & 4269.28 & -2.17 & KX & 12 & -5.70 \\
\hline \multirow[t]{2}{*}{39} & 4539.62 & -2.53 & MF & 8 & -5.44 \\
\hline & 4565.78 & -2.11 & MF & 10 & -5.77 \\
\hline \multirow[t]{7}{*}{44} & 4555.02 & -1.38 & MF & 33 & -5.72 \\
\hline & 4558.66 & -0.66 & MF & 60 & -5.55 \\
\hline & 4588.22 & -0.63 & MF & 58 & -5.67 \\
\hline & 4592.09 & -1.22 & MF & 17 & -6.34 \\
\hline & 4616.64 & -1.29 & MF & 25 & -6.04 \\
\hline & 4618.82 & -1.11 & MF & 32 & -6.00 \\
\hline & 4634.10 & -1.24 & MF & 30 & -5.93 \\
\hline 161 & 4195.41 & -2.32 & KX & 6 & -5.34 \\
\hline \multirow[t]{2}{*}{162} & 4145.77 & -1.16 & $\mathrm{KX}$ & 19 & -5.75 \\
\hline & 4224.85 & -1.73 & KX & 12 & -5.46 \\
\hline 165 & 4082.30 & -1.23 & KX & 7 & -6.23 \\
\hline 177 & 4697.61 & -1.88 & MF & 5 & -5.62 \\
\hline 190 & 4901.62 & -0.83 & KX & 10 & -5.88 \\
\hline 193 & 4070.90 & -0.75 & $\mathrm{KX}$ & 8 & -6.12 \\
\hline 194 & 4003.33 & -0.60 & KX & 8 & -6.28 \\
\hline- & 4254.56 & -1.75 & KX & 6 & -5.52 \\
\hline \multicolumn{3}{|c|}{ Mn I } & & \multicolumn{2}{|c|}{$\left(\log \mathrm{Mn} / N_{T}=-4.31\right)$} \\
\hline 3 & 4030.76 & -0.47 & $\mathrm{MF}$ & 23 & -4.31 \\
\hline \multicolumn{3}{|c|}{ Mn II } & \multicolumn{3}{|c|}{$\left(\log \mathrm{Mn} / N_{T}=-4.85 \pm 0.32\right)$} \\
\hline \multirow[t]{30}{*}{-} & 3844.17 & -1.38 & $\mathrm{KX}$ & 35 & -4.98 \\
\hline & 3917.32 & -1.15 & KX & 15 & -5.21 \\
\hline & 3941.22 & -2.62 & KX & 14 & -4.49 \\
\hline & 3943.85 & -2.46 & KX & 20 & -4.45 \\
\hline & 4000.04 & -1.21 & KX & 16 & -4.73 \\
\hline & 4081.45 & -2.24 & $\mathrm{KX}$ & 18 & -4.39 \\
\hline & 4083.66 & -4.82 & KX & 5 & -4.53 \\
\hline & 4105.00 & -1.35 & $\mathrm{KX}$ & 12 & -5.52 \\
\hline & 4110.62 & -1.51 & $\mathrm{KX}$ & 38 & -4.49 \\
\hline & 4136.94 & -1.29 & $\mathrm{KX}$ & 32 & -4.87 \\
\hline & 4140.44 & -2.45 & $\mathrm{KX}$ & 8 & -4.91 \\
\hline & 4171.51 & -2.11 & KX & 15 & -4.58 \\
\hline & 4184.47 & -1.95 & $\mathrm{KX}$ & 12 & -4.89 \\
\hline & 4200.28 & -1.74 & $\mathrm{KX}$ & 9 & -5.25 \\
\hline & 4205.39 & -3.38 & $\mathrm{KX}$ & 26 & -5.05 \\
\hline & 4206.37 & -1.57 & KX & 28 & -5.08 \\
\hline & 4237.87 & -2.96 & KX & 5 & -4.67 \\
\hline & 4239.19 & -2.25 & $\mathrm{KX}$ & 18 & -4.72 \\
\hline & 4240.39 & -2.07 & KX & 15 & -4.65 \\
\hline & 4242.92 & -2.99 & KX & 8 & -4.47 \\
\hline & 4244.24 & -2.40 & KX & 17 & -4.62 \\
\hline & 4251.74 & -1.06 & $\mathrm{KX}$ & 34 & -5.04 \\
\hline & 4252.96 & -1.14 & $\mathrm{KX}$ & 55 & -4.27 \\
\hline & 4259.20 & -1.59 & KX & 37 & -4.78 \\
\hline & 4260.46 & -4.25 & KX & 12 & -4.65 \\
\hline & 4281.94 & -2.55 & $\mathrm{KX}$ & 16 & -4.51 \\
\hline & 4282.47 & -1.68 & $\mathrm{KX}$ & 16 & -5.28 \\
\hline & 4283.77 & -2.20 & $\mathrm{KX}$ & 13 & -4.96 \\
\hline & 4288.07 & -2.76 & $\mathrm{KX}$ & 6 & -4.78 \\
\hline & 4292.25 & -2.23 & $\mathrm{KX}$ & 21 & -4.65 \\
\hline
\end{tabular}


Table 6. continued

\begin{tabular}{|c|c|c|c|c|c|}
\hline mult. & $\lambda(\ddot{A})$ & $\log g f$ & Ref. & $W_{\lambda}(\mathrm{m} \AA)$ & $\log N / N_{T}$ \\
\hline \multicolumn{6}{|c|}{ Mn II (cont.) } \\
\hline \multirow[t]{27}{*}{ - } & 4310.70 & -0.16 & KX & 5 & -5.06 \\
\hline & 4326.63 & -1.25 & $\mathrm{KX}$ & 62 & -4.32 \\
\hline & 4328.80 & -3.63 & $\mathrm{KX}$ & 30 & -4.70 \\
\hline & 4343.98 & -1.09 & $\mathrm{KX}$ & 53 & -4.77 \\
\hline & 4348.39 & -1.50 & KX & 21 & -5.35 \\
\hline & 4363.25 & -1.91 & $\mathrm{KX}$ & 13 & -5.17 \\
\hline & 4365.22 & -1.35 & KX & 10 & -5.38 \\
\hline & 4377.74 & -2.14 & $\mathrm{KX}$ & 10 & -5.12 \\
\hline & 4379.61 & -1.85 & $\mathrm{KX}$ & 10 & -5.44 \\
\hline & 4478.64 & -0.95 & KX & 19 & -5.38 \\
\hline & 4689.56 & -2.54 & $\mathrm{KX}$ & 9 & -4.49 \\
\hline & 4717.26 & -1.86 & $\mathrm{KX}$ & 7 & -5.10 \\
\hline & 4727.84 & -2.02 & KX & 15 & -5.05 \\
\hline & 4730.40 & -2.15 & KX & 23 & -4.66 \\
\hline & 4742.95 & -2.98 & KX & 7 & -4.49 \\
\hline & 4738.30 & -2.24 & $\mathrm{KX}$ & 32 & -4.29 \\
\hline & 4755.73 & -1.24 & KX & 45 & -4.87 \\
\hline & 4764.73 & -1.35 & $\mathrm{KX}$ & 45 & -4.76 \\
\hline & 4791.78 & -1.72 & $\mathrm{KX}$ & 20 & -4.80 \\
\hline & 4806.82 & -1.56 & $\mathrm{KX}$ & 26 & -5.13 \\
\hline & 4811.62 & -2.34 & $\mathrm{KX}$ & 6 & -5.22 \\
\hline & 4830.06 & -1.85 & KX & 12 & -4.96 \\
\hline & 4839.74 & -1.86 & $\mathrm{KX}$ & 15 & -4.81 \\
\hline & 4842.32 & -2.00 & KX & 10 & -4.94 \\
\hline & 4847.61 & -1.81 & KX & 10 & -5.48 \\
\hline & 4920.44 & -2.09 & KX & 23 & -4.66 \\
\hline & 4921.23 & -1.58 & $\mathrm{KX}$ & 18 & -4.76 \\
\hline Fe I & & & $(\log$ & $\mathrm{e} / N_{T}=-$ & $44 \pm 0.13)$ \\
\hline 41 & 4383.54 & +0.20 & $\mathrm{MF}$ & 11 & -4.56 \\
\hline 42 & 4271.76 & -0.16 & $\mathrm{MF}$ & 10 & -4.23 \\
\hline \multirow[t]{3}{*}{43} & 4045.82 & +0.28 & $\mathrm{MF}$ & 13 & -4.54 \\
\hline & 4063.59 & +0.07 & $\mathrm{MF}$ & 11 & -4.40 \\
\hline & 4071.74 & -0.02 & $\mathrm{MF}$ & 7 & -4.47 \\
\hline \multicolumn{3}{|c|}{ Fe II } & \multicolumn{3}{|c|}{$\left(\log \mathrm{Fe} / N_{T}=-4.59 \pm 0.19\right)$} \\
\hline \multirow[t]{2}{*}{3} & 3938.29 & -3.89 & $\mathrm{MF}$ & 18 & -4.53 \\
\hline & 3945.21 & -4.19 & MF & 5 & -4.87 \\
\hline 22 & 4124.79 & -4.20 & MF & 5 & -4.52 \\
\hline 25 & 4670.17 & -3.97 & KX & 5 & -4.68 \\
\hline 26 & 4580.06 & -3.65 & KX & 10 & -4.65 \\
\hline \multirow[t]{4}{*}{27} & 4128.74 & -3.77 & MF & 17 & -4.26 \\
\hline & 4273.32 & -3.34 & MF & 19 & -4.55 \\
\hline & 4385.38 & -2.57 & MF & 31 & -4.91 \\
\hline & 4416.82 & -2.60 & MF & 28 & -4.96 \\
\hline \multirow[t]{3}{*}{28} & 4258.16 & -3.40 & MF & 20 & -4.45 \\
\hline & 4296.57 & -3.01 & MF & 12 & -5.16 \\
\hline & 4369.40 & -3.67 & MF & 7 & -4.72 \\
\hline 29 & 4002.08 & -3.37 & KX & 8 & -4.98 \\
\hline \multirow[t]{2}{*}{32} & 4314.29 & -3.49 & KX & 24 & -4.24 \\
\hline & 4384.33 & -3.50 & MF & 14 & -4.59 \\
\hline 36 & 4993.35 & -3.65 & $\mathrm{MF}$ & 10 & -4.55 \\
\hline \multirow[t]{7}{*}{37} & 4472.92 & -3.43 & $\mathrm{MF}$ & 11 & -4.72 \\
\hline & 4515.34 & -2.48 & $\mathrm{MF}$ & 49 & -4.40 \\
\hline & 4520.22 & -2.60 & $\mathrm{MF}$ & 45 & -4.42 \\
\hline & 4555.89 & -2.29 & MF & 53 & -4.46 \\
\hline & 4582.84 & -3.10 & $\mathrm{MF}$ & 17 & -4.80 \\
\hline & 4629.34 & -2.37 & $\mathrm{MF}$ & 58 & -4.23 \\
\hline & 4663.70 & -4.28 & KX & 4 & -4.32 \\
\hline \multirow[t]{2}{*}{38} & 4541.52 & -3.05 & $\mathrm{MF}$ & 28 & -4.47 \\
\hline & 4576.33 & -3.04 & $\mathrm{MF}$ & 21 & -4.72 \\
\hline
\end{tabular}

Table 6. continued

\begin{tabular}{|c|c|c|c|c|c|}
\hline mult. & $\lambda(\AA)$ & $\log g f$ & Ref. & $W_{\lambda}(\mathrm{m} \AA)$ & $\log N / N_{T}$ \\
\hline \multicolumn{6}{|c|}{ Fe II (cont.) } \\
\hline \multirow[t]{2}{*}{38} & 4583.83 & -2.02 & $\mathrm{MF}$ & 51 & -4.83 \\
\hline & 4620.51 & -3.28 & $\mathrm{MF}$ & 10 & -4.92 \\
\hline 42 & 4923.93 & -1.32 & $\mathrm{MF}$ & 89 & -4.16 \\
\hline 43 & 4731.44 & -3.36 & MF & 23 & -4.30 \\
\hline 126 & 4032.95 & -2.83 & KX & 15 & -4.35 \\
\hline 127 & 4024.55 & -2.48 & $\mathrm{MF}$ & 35 & -4.05 \\
\hline 128 & 3845.18 & -2.26 & KX & 20 & -4.75 \\
\hline 168 & 4953.98 & -2.76 & KX & 12 & -4.02 \\
\hline 172 & 4044.01 & -2.36 & KX & 6 & -4.77 \\
\hline 173 & 3935.94 & -1.86 & MF & 26 & -4.46 \\
\hline 173 & 3906.04 & -1.83 & $\mathrm{MF}$ & 22 & -4.61 \\
\hline \multirow[t]{2}{*}{186} & 4625.91 & -2.22 & KX & 6 & -4.76 \\
\hline & 4635.33 & -1.65 & MF & 23 & -4.53 \\
\hline 190 & 3938.97 & -1.85 & MF & 15 & -4.69 \\
\hline 212 & 4057.46 & -1.54 & KX & 11 & -4.54 \\
\hline \multirow[t]{37}{*}{-} & 4357.58 & -2.10 & KX & 10 & -4.58 \\
\hline & 4451.54 & -1.82 & $\mathrm{KX}$ & 13 & -4.67 \\
\hline & 4596.02 & -1.82 & KX & 15 & -4.55 \\
\hline & 4455.26 & -1.99 & KX & 8 & -4.69 \\
\hline & 4480.69 & -2.34 & KX & 11 & -4.19 \\
\hline & 4579.52 & -2.36 & KX & 8 & -4.35 \\
\hline & 4820.83 & -0.69 & $\mathrm{KX}$ & 7 & -4.22 \\
\hline & 4826.68 & -0.44 & $\mathrm{KX}$ & 4 & -4.76 \\
\hline & 4908.15 & -0.30 & KX & 7 & -4.58 \\
\hline & 4913.29 & +0.01 & KX & 16 & -4.43 \\
\hline & 4948.10 & -0.32 & $\mathrm{KX}$ & 7 & -4.56 \\
\hline & 4948.79 & -0.01 & KX & 9 & -4.71 \\
\hline & 4951.58 & +0.18 & KX & 15 & -4.64 \\
\hline & 4977.03 & +0.04 & KX & 8 & -4.80 \\
\hline & 4984.48 & +0.01 & KX & 11 & -4.62 \\
\hline & 4990.51 & +0.18 & $\mathrm{KX}$ & 8 & -4.99 \\
\hline & 4999.18 & -0.48 & KX & 6 & -4.49 \\
\hline & 5001.96 & +0.90 & $\mathrm{KX}$ & 46 & -4.27 \\
\hline & 5004.20 & +0.50 & KX & 30 & -4.40 \\
\hline & 5006.80 & -0.43 & KX & 8 & -4.34 \\
\hline & 5019.46 & -2.70 & $\mathrm{KX}$ & 14 & -3.99 \\
\hline & 5030.63 & +0.40 & KX & 17 & -4.75 \\
\hline & 5035.71 & +0.61 & KX & 13 & -5.14 \\
\hline & 5045.11 & -0.13 & KX & 13 & -4.39 \\
\hline & 5047.64 & -0.07 & KX & 10 & -4.62 \\
\hline & 5060.26 & -0.52 & $\mathrm{KX}$ & 6 & -4.36 \\
\hline & 5061.72 & +0.22 & $\mathrm{KX}$ & 19 & -4.48 \\
\hline & 5067.89 & -0.20 & $\mathrm{KX}$ & 12 & -4.35 \\
\hline & 5070.90 & +0.24 & KX & 18 & -4.56 \\
\hline & 5074.05 & -1.97 & KX & 7 & -4.53 \\
\hline & 5075.76 & +0.28 & $\mathrm{KX}$ & 13 & -4.76 \\
\hline & 5082.23 & -0.10 & KX & 8 & -4.66 \\
\hline & 5086.31 & -0.48 & KX & 7 & -4.33 \\
\hline & 5089.21 & -0.04 & KX & 10 & -4.61 \\
\hline & 5094.91 & -0.56 & KX & 9 & -4.13 \\
\hline & 5093.58 & +0.11 & KX & 23 & -4.19 \\
\hline & 5097.27 & +0.31 & KX & 18 & -4.60 \\
\hline \multicolumn{4}{|c|}{ Fe III } & \multicolumn{2}{|c|}{$\left(\log \mathrm{Fe} / N_{T}=-4.86\right)$} \\
\hline 4 & 4419.59 & -2.22 & KX & 5 & -4.86 \\
\hline \multicolumn{4}{|l|}{$\mathrm{Ni}$ II } & \multicolumn{2}{|c|}{$\left(\log \mathrm{Ni} / N_{T}=-6.46\right)$} \\
\hline 11 & 4067.05 & -1.83 & KX & 12 & -6.46 \\
\hline \multicolumn{3}{|c|}{ Ga II } & \multicolumn{3}{|c|}{$\left(\log \mathrm{Ni} / N_{T}=-5.94 \pm 0.13\right)$} \\
\hline- & 4251.15 & +0.46 & JS & 14 & -5.85 \\
\hline & 4255.72 & +0.68 & JS & 14 & -6.03 \\
\hline
\end{tabular}


Table 6. continued

\begin{tabular}{cccccc}
\hline mult. & $\lambda(\AA)$ & $\log g f$ & Ref. & $W_{\lambda}(\mathrm{mA})$ & $\log \mathrm{N} / N_{T}$ \\
\hline Sr II & & & \multicolumn{2}{c}{$\left.\log \mathrm{Sr} / N_{T}=-8.74 \pm 0.39\right)$} \\
1 & 4077.71 & +0.15 & $\mathrm{WM}$ & 9 & -9.02 \\
& 4215.52 & -0.17 & $\mathrm{WM}$ & 14 & -8.47 \\
Y II & & & & $\left(\log \mathrm{Y} / N_{T}=-8.13\right)$ \\
22 & 4883.68 & +0.07 & $\mathrm{HL}$ & 7 & -8.13 \\
Zr II & & & $\left(\log \mathrm{Zr} / N_{T}=-7.72 \pm 0.27\right)$ \\
17 & 3915.94 & -0.77 & $\mathrm{BG}$ & 7 & -7.52 \\
19 & 4156.24 & -0.71 & $\mathrm{~GB}$ & 5 & -7.74 \\
41 & 4149.22 & -0.03 & $\mathrm{~GB}$ & 8 & -8.09 \\
42 & 4161.20 & -0.58 & $\mathrm{~GB}$ & 9 & -7.52 \\
Ce II & & & & $\left(\log \mathrm{Ce} / N_{T}=-6.73\right)$ \\
2 & 4460.21 & +0.17 & $\mathrm{KX}$ & 16 & -6.73 \\
Hg II & & & & $\left(\log \mathrm{Hg} / N_{T}=-6.53\right)$ \\
- & 3983.96 & -1.73 & $\mathrm{DW}$ & 57 & -6.53 \\
& & & & \multicolumn{3}{c}{} \\
\hline
\end{tabular}

$g f$ values References:

$\mathrm{BG}=$ Biemont et al. (1981)

DW $=$ Dworetsky $(1980)$

$\mathrm{GB}=$ Grevesse et al. (1981)

$\mathrm{HL}=$ Hannaford et al. (1982)

JS = Jugaku et al. (1961)

KX $=$ Kurucz (1995)

$\mathrm{LA}=$ Lanz \& Artru (1985)

MF = Martin et al. (1988) and Fuhr et al. (1988)

$\mathrm{WM}=$ Wiese \& Martin (1980)

WS $=$ Wiese et al. (1966, 1969) 